\title{
Some properties of filters in Stonean residuated lattices
}

\author{
Holdon Liviu-Constantin \\ Faculty of Exact Sciences, Department of Mathematics, University of Craiova 13, Al. I.Cuza st., 200585, Craiova, \\ Romania
}

\begin{abstract}
The goal of this paper is to extend the study of Stonean residuated lattices ([8]), we introduce the notions of i-filter's radical and boolean i-filter in Stonean residuated lattices and we put in evidence some specific properties of those .
\end{abstract}

Keywords: Residuated lattice, i-filter, prime i-filter, maximal i-filter

2010 MSC: 03G10, 03G25, 06D05, 06D35, 08A72.

\section{INTRODUCTION}

Let $L$ be a lattice with 0 . We recall ([1],[2]) that an element $a \in L$ is pseudocomplemented if there is a largest member of $L$ which is disjoint with $a$. If such an element exists, it is denoted by $a^{*}$ and is called pseudocomplement of $a$ (that is, $a^{*}=\max \{x \in L: a \wedge x=0\}$ ).

A pseudocomplemented lattice is a lattice with 0 in which every element has a pseudocomplement. Clearly, a pseudocomplemented lattice is bounded, where $1=0^{*}$.

If $(L, \wedge, \vee, 0,1)$ is a bounded lattice, we recall ([1],[3],[7],[21]) that an element $a \in L$ is called complemented if there is an element $b \in L$ such that $a \wedge b=0$ and $a \vee b=1$; if such an element exists it is called a complement of $a$. Complements are, in general, not unique, unless the lattice is distributive (see Lemma 2.6.2, [3]). In residuated lattices ([21]) the complements are unique, although the underlying lattice need not be distributive ([9]).

A boolean element of a residuated lattice $L$ is a complemented element of the underlying lattice of $L$. It is proved in [21] that for a boolean element $e \in L$, its complement is $e^{*}=e \rightarrow 0$. In the same paper is proved that the set of boolean elements of $L$ form a subalgebra of $L$, that is a boolean algebra denoted by $B(L)$, and that $e \in L$ belongs to $B(L)$ if, and only if, $e \vee e^{*}=1$. These results are also presented in [3], [7] and [24]. For a residuated lattice $L$, the boolean subalgebra $B(L)$ is called the boolean center of $L$.

In some situations (see [1],[2]) $L$ is supposed to be distributive.

A pseudocomplemented lattice $L$ is called Stonean lattice if $x^{*} \vee x^{* *}=1$ for every $x \in L$. In [1] is given the following theorem of characterization for Stonean distributive lattices:

Theorem 1 For a pseudocomplemented distributive lattice $L$ with 0 , the following are equivalent:

(i) $L$ is a Stonean lattice;

(ii) $(x \wedge y)^{*}=x^{*} \vee y^{*}$ for every $x, y \in L$;

(iii) $(x \vee y)^{* *}=x^{* *} \vee y^{* *}$ for every $x, y \in L$;

(iv) Every prime ideal in $L$ contains at most one minimal prime ideal;

(v) If $I$ and $J$ are distinct minimal prime ideals in $L$, then $I \vee J=L$.

The study of pseudocomplemented distributive lattices commenced with a paper by V. Glivenko in 1929 (see [15]). Although in the 1937 classic paper of M. H. Stone ([25]) there is a reference to what we now call Stone algebras, there were G. Grätzer and E. T. Schmidt ([16]) who first solved a problem of M. H. Stone and thereby generated widespread interest in the topic. G. Birkhoff [[2], 1948 edition], in fact, was the first paper in which the term Stonean lattice is used.

We recall that a residuated lattice ([14],[28]) is an algebra $(L, \vee, \wedge, \otimes, \rightarrow, 0,1)$ of type $(2,2,2,2,00)$ equipped with an order $\leq$ such that 
$L R_{1}:(L, \vee, \wedge, 0,1)$ is a bounded lattice relative to $\leq$;

$L R_{2}:(L, \otimes, 1)$ is a commutative ordered monoid;

$L R_{3}: \otimes$ and $\rightarrow$ form an adjoint pair, i.e., $a \otimes x \leq b$ iff $x \leq a \rightarrow b$, for all $a, b, x \in L$.

For examples of residuated lattices see [6],[7],[13],[14],[19],[24],[26].

In what follows (unless otherwise specified) by $L$ we denote a residuated lattice.

For $x \in L$ we define $x^{*}=x \rightarrow 0$ and $x^{* *}=\left(x^{*}\right)^{*}$.

Taking as a guide line the case of lattices with 0 , the residuated lattice $L$ is called Stonean ([11],[12],[20],[27]) if $x^{*} \vee x^{* *}=1$ for every $x \in L$.

We recall ([19],[24]) that in general residuated lattices need not be distributive.

Every Stonean residuated lattice ([8]) $L$ is pseudocomplemented, where for $x \in L, x^{*}$ is the pseudocomplement of $x$.

In the absence of distributivity of $L$, it is possible that Theorem 1 be not true in the case of $L$. Indeed, in [8] we put in evidence some properties of i-ideals in a Stonean residuated lattice $L$, in connection with properties (ii) and (iv) (for the dual form) from Theorem 1.

Residuated lattices and Stone algebras are two topics much studied in lattice theory, with impact on logic. One of the topics studied in residuated lattices is the theory of the so-called implicative ideals and filters. This paper deals with algebras that are both residuated lattices and Stone algebras, the subject-matter is the detection of properties of some special types of implicative filters (i-filters for short) that are specific to Stonean residuated lattices. We mention that we introduce the notions of i-filter's radical and boolean i-filter in Stonean ressiduated lattices. Several interesting results are obtained.

\section{PRELIMINARIES}

For $x \in L$ and $n \geq 1$ we define $x^{*}=x \rightarrow 0, \quad x^{* *}=\left(x^{*}\right)^{*}, x^{0}=1$ and $x^{n}=x^{n-1} \otimes x$ for $n \geq 1$.

Proposition 1 Let $L$ be a residuated lattice. Then for every $x, y, z \in L$, we have:

$\left(\mathrm{c}_{1}\right) x \otimes y \leq x, y$, so $x \otimes y \leq x \wedge y$;

(c $\left.c_{2}\right) x \leq y \Leftrightarrow x \rightarrow y=1$, and $y \leq x \rightarrow y$;

(c $\left.c_{3}\right)$ If $x \leq y$, then $x \otimes z \leq y \otimes z$;

(c) $x \leq y \Rightarrow z \rightarrow x \leq z \rightarrow y$, and $x \leq y \Rightarrow y \rightarrow z \leq x \rightarrow z$;

(c $\left.\mathrm{c}_{5}\right) x \rightarrow(y \rightarrow z)=(x \otimes y) \rightarrow z$, so $x \rightarrow(y \rightarrow z)=y \rightarrow(x \rightarrow z)$;

$\left(\mathrm{c}_{6}\right) \quad x \otimes(y \vee z)=(x \otimes y) \vee(x \otimes z)$, and $x \otimes(y \wedge z) \leq(x \otimes y) \wedge(x \otimes z)$

$(x \rightarrow z) \wedge(y \rightarrow z)=(x \vee y) \rightarrow z$, and $\quad(x \rightarrow z) \vee(y \rightarrow z) \leq(x \wedge y) \rightarrow z$,
$\left(\mathrm{c}_{7}\right)$
$x \rightarrow(y \wedge z)=(x \rightarrow y) \wedge(x \rightarrow z) ;$

(c 8$) x \vee(y \otimes z) \geq(x \vee y) \otimes(x \vee z)$

$\left(c_{9}\right)(x \vee y)^{*}=x^{*} \wedge y^{*},\left(x \rightarrow y^{* *}\right)^{* *}=x \rightarrow y^{* *}$, and $x^{* *} \rightarrow y^{* *}=y^{*} \rightarrow x^{*}=x \rightarrow y^{* *}$;

$\left(\mathrm{c}_{10}\right) x \otimes x^{*}=0,1^{*}=0,0^{*}=1, x \leq x^{* *}, x \rightarrow y \leq y^{*} \rightarrow x^{*}$, and $x^{* * *}=x^{*}$.

\section{Consider the following identities:}




$$
\begin{aligned}
& x \wedge y=x \otimes(x \rightarrow y) \quad(\text { divisibility) }, \\
& (x \rightarrow y) \vee(y \rightarrow x)=1 \quad \text { (pre-linearity), } \\
& x \otimes y=x \wedge y \quad \text { (idempotence). }
\end{aligned}
$$

Definition 1 A residuated lattice $L$ is called:

(i) Divisible if $L$ verifies (11),

(ii) MTL-algebra if $L$ verifies (12),

(iii) BL-algebra if $L$ verifies (11) and (12),

(iv) G-algebra if $L$ is a BL-algebra and verifies (13).

We present some examples of residuated lattices that we will use in the sequel, for illustrating various properties and various classes of residuated lattices.

Example 1. Let $L=\{0, a, b, c, 1\}$ with $0<a, b<c<1$, but $a$ and $b$ are incomparable.

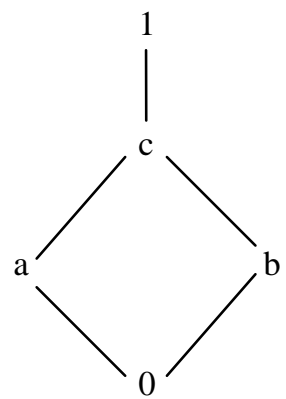

Then $(L, \wedge, \vee, \otimes, \rightarrow, 0,1)$ is a residuated lattice ([19], page 187), where $\rightarrow$ and $\otimes$ are defined as in the tables:

$\begin{array}{llllllllllll}\rightarrow & 0 & a & b & c & 1 & \otimes & 0 & a & b & c & 1 \\ 0 & 1 & 1 & 1 & 1 & 1 & 0 & 0 & 0 & 0 & 0 & 0 \\ a & b & 1 & b & 1 & 1 & a & 0 & a & 0 & a & a \\ b & a & a & 1 & 1 & 1 & b & 0 & 0 & b & b & b \\ c & 0 & a & b & 1 & 1 & c & 0 & a & b & c & c \\ 1 & 0 & a & b & c & 1 & 1 & 0 & a & b & c & 1\end{array}$

Example 2. $L=\{0, a, b, c, d, e, f, g, 1\}$ with $0<a<b<e<1,0<a<d<e<1$, $0<a<d<g<1,0<c<d<e<1,0<c<d<g<1,0<c<f<g<1$ and elements $\{a, c\}$, $\{b, d\},\{d, f\},\{e, g\}$ and $\{b, f\}$ are pairwise incomparable. 


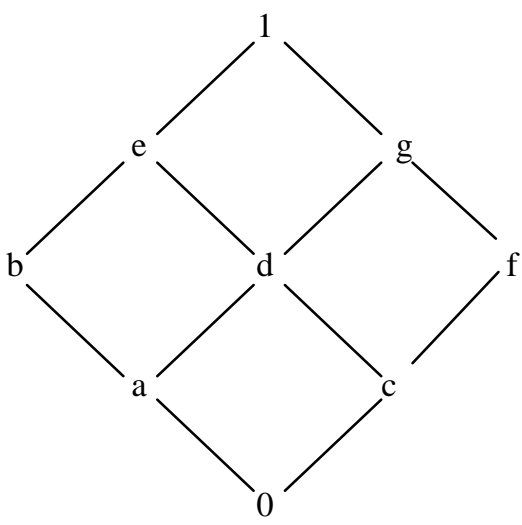

Then ([19], page 166) $L$ becomes a residuated lattice relative to the following operations:

$\begin{array}{llllllllll}\rightarrow & 0 & a & b & c & d & e & f & g & 1 \\ 0 & 1 & 1 & 1 & 1 & 1 & 1 & 1 & 1 & 1 \\ a & g & 1 & 1 & g & 1 & 1 & g & 1 & 1 \\ b & f & g & 1 & f & g & 1 & f & g & 1 \\ c & e & e & e & 1 & 1 & 1 & 1 & 1 & 1 \\ d & d & e & e & g & 1 & 1 & g & 1 & 1 \\ e & c & d & e & f & g & 1 & f & g & 1 \\ f & b & b & b & e & e & e & 1 & 1 & 1 \\ g & a & b & b & d & e & e & g & 1 & 1 \\ 1 & 0 & a & b & c & d & e & f & g & 1\end{array}$

$\begin{array}{llllllllll}\otimes & 0 & a & b & c & d & e & f & g & 1 \\ 0 & 0 & 0 & 0 & 0 & 0 & 0 & 0 & 0 & 0 \\ a & 0 & 0 & a & 0 & 0 & a & 0 & 0 & a \\ b & 0 & a & b & 0 & a & b & 0 & a & b \\ c & 0 & 0 & 0 & 0 & 0 & 0 & c & c & c \\ d & 0 & 0 & a & 0 & 0 & a & c & c & d \\ e & 0 & a & b & 0 & a & b & c & d & e \\ f & 0 & 0 & 0 & c & c & c & f & f & f \\ g & 0 & 0 & a & c & c & d & f & f & g \\ 1 & 0 & a & b & c & d & e & f & g & 1\end{array}$

Example 3. $L=\{0, a, b, c, d, e, f, g, 1\}$ with $0<a<b<e<1,0<a<d<e<1$, $0<a<d<g<1,0<c<d<e<1,0<c<d<g<1,0<c<f<g<1$ and elements $\{a, c\}$, $\{b, d\},\{d, f\},\{e, g\}$ and $\{b, f\}$ are pairwise incomparable (see the diagram from Example 2).

Then ([23]) $L$ becomes a residuated lattice relative to the following operations:

$\begin{array}{llllllllll}\rightarrow & 0 & a & b & c & d & e & f & g & 1 \\ 0 & 1 & 1 & 1 & 1 & 1 & 1 & 1 & 1 & 1 \\ a & f & 1 & 1 & f & 1 & 1 & f & 1 & 1 \\ b & f & g & 1 & f & g & 1 & f & g & 1 \\ c & b & b & b & 1 & 1 & 1 & 1 & 1 & 1 \\ d & 0 & b & b & f & 1 & 1 & f & 1 & 1 \\ e & 0 & a & b & f & g & 1 & f & g & 1 \\ f & b & b & b & e & e & e & 1 & 1 & 1 \\ g & 0 & b & b & c & e & e & f & 1 & 1 \\ 1 & 0 & a & b & c & d & e & f & g & 1\end{array}$

$\begin{array}{llllllllll}\otimes & 0 & a & b & c & d & e & f & g & 1 \\ 0 & 0 & 0 & 0 & 0 & 0 & 0 & 0 & 0 & 0 \\ a & 0 & a & a & 0 & a & a & 0 & a & a \\ b & 0 & a & b & 0 & a & b & 0 & a & b \\ c & 0 & 0 & 0 & c & c & c & c & c & c \\ d & 0 & a & a & c & d & d & c & d & d \\ e & 0 & a & b & c & d & e & c & d & e \\ f & 0 & 0 & 0 & c & c & c & f & f & f \\ g & 0 & a & a & c & d & d & f & g & g \\ 1 & 0 & a & b & c & d & e & f & g & 1\end{array}$

Example 4 Let $L=\{0, a, b, n, c, d, 1\}$ with $0<a<n<d<1,0<b<n<c<1$, but $(a, b)$ and $(c, d)$ are pairwise incomparable. 


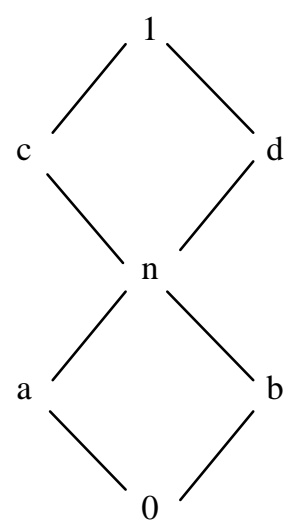

Then ([19], page 191) $L$ becomes a distributive residuated lattice relative to the operations:

$\begin{array}{llllllllllllllll}\rightarrow & \mathrm{O} & a & b & n & c & d & 1 & \otimes & \mathrm{O} & a & b & n & c & d & 1 \\ \mathrm{O} & 1 & 1 & 1 & 1 & 1 & 1 & 1 & \mathrm{O} & \mathrm{O} & \mathrm{O} & \mathrm{O} & \mathrm{O} & \mathrm{O} & \mathrm{O} & \mathrm{O} \\ a & \mathrm{~b} & 1 & b & 1 & 1 & 1 & 1 & a & \mathrm{O} & a & \mathrm{O} & a & a & a & a \\ b & a & a & 1 & 1 & 1 & 1 & 1 & b & \mathrm{O} & \mathrm{O} & b & b & b & b & b \\ n & \mathrm{O} & a & b & 1 & 1 & 1 & 1 & n & \mathrm{O} & a & b & n & n & n & n \\ c & \mathrm{O} & a & b & d & 1 & d & 1 & c & \mathrm{O} & a & b & n & c & n & c \\ d & \mathrm{O} & a & b & c & c & 1 & 1 & d & \mathrm{O} & a & b & n & n & d & d \\ 1 & \mathrm{O} & a & b & n & c & d & 1 & 1 & \mathrm{O} & a & b & n & c & d & 1\end{array}$

\section{1. i-Filters in residuated lattices}

We say that an ordered set $L$ is a lattice if for every two elements $a, b \in L$ there exist $a \wedge b$ and $a \vee b$ in $L$.

In lattice theory a lattice filter is defined as follows:

Definition 2 A non-empty subset $F$ will be called a lattice filter (filter, for short) of $L$ if

$\left(F_{1}\right)$ If $a \leq b$ and $a \in F$, then $b \in F$;

$\left(F_{2}\right)$ If $a, b \in F$, then $a \wedge b \in F$.

We denote by $\mathrm{F}(L)$ the set of all filters of $L$.

In residuated lattice theory we have the following notions:

Definition 3 An implicative filter ( $i$-filter, for short) is a nonempty subset $F$ of $L$ such that

$\left(F_{1}\right)$ If $x \leq y$ and $x \in F$, then $y \in F$;

$\left(F_{3}\right)$ If $x, y \in F$, then $x \otimes y \in F$.

We denote by $\mathrm{F}_{i}(L)$ the set of all i-filters of $L$.

Remark 1 1. $F$ is an implicative filter of $L$ iff $1 \in F$ and $x, x \rightarrow y \in F$, then $y \in F$ (that is, $F$ is a deductive system of $L$ ).

2. Every i-filter is a filter in the lattice $(L, \wedge, \vee)$, but the converse is not true (see [8],[20]).

So, if we denote by $\mathrm{F}(L)\left(\mathrm{F}_{i}(L)\right)$ the set of all lattice filters (i-filters) of $L$, then $\mathrm{F}_{i}(L) \subseteq \mathrm{F}(L)$.

Remark 2 There are residuated lattices in which lattice filters are not i-filters.

Indeed, we consider the Example 2. Clearly, $F=\{d, e, g, 1\}$ is a lattice filter.

If $F$ is an i-filter, since $d \otimes d=0$, and an i-filter is closed with respect to the operation $\otimes$, then we deduce that $0 \in F$, a contradiction, so, $F$ is not an i-filter.

We have ([6]), $\mathrm{F}_{i}(L)=\mathrm{F}(L)$ iff $x \otimes y=x \wedge y$ for every $x, y \in L$.

For a nonempty subset $S$ of $L$ we denote by $\langle S\rangle$ the i-filter generated by $S$. If $a \in L$, the i-filter generated by $\{a\}$ will be denoted by $\langle a\rangle$ (also, $\langle a\rangle$ is called principal). If $F \in \mathrm{F}_{i}(L)$ and $a \in L \backslash F$, 
then $\langle F \cup\{a\}\rangle$ will be denoted by $F\langle a\rangle$.

Proposition 2 ([6],[7],[24]) Let $L$ be a residuated lattice, $S \subseteq L$ a nonempty subset, $F \in \mathrm{F}_{i}(L)$ and $a \in L \backslash F$.

Then:

(i) $\langle S\rangle=\left\{x \in L: s_{1} \otimes \ldots \otimes s_{n} \leq x\right.$, for some $\left.s_{1}, \ldots, s_{n} \in S\right\}$;

(ii) $\langle a\rangle=\left\{x \in L: a^{n} \leq x\right.$, for some $\left.n \geq 1\right\}$;

(iii) $F\langle a\rangle=\left\{x \in L: a^{n} \rightarrow x \in F\right.$, for some $\left.n \geq 1\right\}$;

(iv) $F\langle a\rangle \cap F\langle b\rangle=F\langle a \vee b\rangle$;

(v) (for every $\left.P \in \mathrm{F}_{i}(L)\right), a \in P$ iff $\langle a\rangle \subseteq P$ iff $a^{n} \in P$, for any $n \geq 1$.

Proposition 3 ([22]) Let $L$ be a residuated lattice and $a, b \in L$. Then:

(i) $a \leq b$ implies $\langle b\rangle \subseteq\langle a\rangle$;

(ii) $\langle a\rangle \vee\langle b\rangle=\langle a \wedge b\rangle=\langle a \otimes b\rangle$;

(iii) $\langle a\rangle \cap\langle b\rangle=\langle a \vee b\rangle$.

Proposition 4 ([6],[7],[24]) For $P \in \mathrm{F}_{i}(L)$ the following are equivalent:

(i) If $P=F_{1} \cap F_{2}$ with $F_{1}, F_{2} \in \mathrm{F}_{i}(L)$, then $P=F_{1}$ or $P=F_{2}$;

(ii) If $F_{1} \cap F_{2} \subseteq P$ with $F_{1}, F_{2} \in \mathrm{F}_{i}(L)$, then $F_{1} \subseteq P$ or $F_{2} \subseteq P$;

(iii) If $x, y \in L$ and $x \vee y \in P$, then $x \in P$ or $y \in P$.

Definition 4 We say that $P \in \mathrm{F}_{i}(L)$ is prime i-filter if $P \neq L$ and $P$ verifies one of the equivalent conditions of Proposition 4. We denote by $\operatorname{Spec}_{i}(L)$ the set of all prime $i$-filters of $L$.

Remark 3 We notice that following Proposition 4, ( $i$ ) in order to prove that an i-filter $P \in \mathrm{F}_{i}(L)$ is not prime it suffices that:

If there exist $x, y \in L$ such that $x \vee y \in P$ with $x \notin P$ and $y \notin P$, then $P$ is not prime.

We recall that an i-filter $M$ of $L$ is called maximal if $M \neq L$ and $M$ is not strictly contained in a proper i-filter of $L$. We denote by $\operatorname{Max}_{i}(L)$ the set of all maximal $i$-filters of $L$.

Remark 4 In any residuated lattice L, maximal i-filters are prime.

Indeed, following Proposition 4, we deduce that every maximal i-filter $M$ of a residuated lattice $L$ is prime because, if there exist two proper i-filters $N, P \in \mathrm{F}_{i}(L)$ such that $M=N \cap P$, then $M \subseteq N$ and $M \subseteq P$, by the maximality of $M$ we deduce that $M=N=P$, that is, $M$ is an inf-irreducible, so prime element in the lattice of i-filters $\left(\mathrm{F}_{i}(L), \subseteq\right)$ of $L$ (by the distributivity of the lattice of i-filters $\left(\mathrm{F}_{i}(L), \subseteq\right)$ of $L$ )

In literature for maximal i-filters in any residuated lattice we have the following characterizations:

Corollary 1 ([4],[7]) For a residuated lattice $(L, \wedge, \vee, \rightarrow, \otimes, 0,1)$ and $M$ a proper $i$-filter of $L$ we have the following equivalent assertions:

(i) $M \in \operatorname{Max}_{i}(L)$

(ii) For any $x \notin M$ there exist $d \in M, n \geq 1$ such that $d \otimes x^{n}=0$;

(iii) For any $x \in L, x \notin M$ iff $\left(x^{n}\right)^{*} \in M$, for some $n \geq 1$; 
(iv) For any $x \notin M$ there is $n \geq 1$ such that $x^{n} \rightarrow y \in M$, for every $y \in L$.

Remark 5 ([7]) If $M$ is a maximal i-filter of a residuated lattice $L$, then $x \in M$ iff $x^{* *} \in M$.

Definition 5 The intersection of the maximal i-filters of a residuated lattice $L$ is called the radical of $L$ and will be denoted by $\operatorname{Rad}(L)$.

\section{Proposition 5 ([13])}

$\operatorname{Rad}(L)=\left\{x \in L\right.$ : for every $n \geq 1$ there is $k_{n} \geq 1$ such that $\left.\left[\left(x^{n}\right)^{*}\right]^{k_{n}}=0\right\}$.

\section{1. i-Filter's radical in Stonean residuated lattices}

Following the papers [8], we may define an additive operation $\oplus$ on any residuated lattice $L$ by setting for all $x, y \in L$,

$$
x \oplus y=\left(x^{*} \otimes y^{*}\right)^{*} .
$$

Clearly, following $\left(\mathrm{c}_{5}\right)$ for all $x, y \in L$, the equation (14) is equivalent with

$$
x \oplus y=x^{*} \rightarrow y^{* *}=y^{*} \rightarrow x^{* *} .
$$

In what follows we will establish the properties of operation $\oplus$ in any residuated lattice.

Proposition 6 ([8]) In any residuated lattice $L$, the operation (14) has the following properties:

(i) $0=0 \oplus 0, x \oplus y=y \oplus x$;

(ii) $(x \oplus y)^{* *}=x \oplus y=x^{* *} \oplus y^{* *}$;

(iii) $x \oplus 0=0 \oplus x=x^{* *}$;

(iv) $x \oplus(y \oplus z)=(x \oplus y) \oplus z$;

(v) $x \oplus 1=1 \oplus x=0^{*}=1$;

(vi) $x \vee y=1 \Rightarrow x \oplus y=1$;

(vii) $x \oplus(y \vee z)^{*}=\left(x \oplus y^{*}\right) \wedge\left(x \oplus z^{*}\right)$;

(viii) $x \oplus\left(y^{*} \vee z^{*}\right)^{*}=(x \oplus y) \wedge(x \oplus z)$;

(ix) $x \oplus x^{*}=x^{*} \oplus x=1$;

( $x) \quad\left(x^{*} \oplus y\right)^{*} \oplus y=\left(y^{*} \oplus x\right)^{*} \oplus x$ is equivalent with

$\left(x^{* * *} \rightarrow y^{* * *}\right)^{* *} \rightarrow y^{* * *}=\left(y^{* *} \rightarrow x^{* * *}\right)^{* *} \rightarrow x^{* * *}$, for all $x, y, z \in L$.

Proof. (i). $0 \oplus 0=\left(0^{*} \otimes 0^{*}\right)^{*}=1^{*}=0, x \oplus y=\left(x^{*} \otimes y^{*}\right)^{*} \stackrel{\left(L r_{2}\right)}{=}\left(y^{*} \otimes x^{*}\right)^{*}=y \oplus x$.

(ii). $(x \oplus y)^{* *}=\left(x^{*} \otimes y^{*}\right)^{* * *} \stackrel{(C 10)}{=}\left(x^{*} \otimes y^{*}\right)^{*}=x \oplus y=\left[\left(x^{* *}\right)^{*} \otimes\left(y^{* *}\right)^{*}\right]^{*}=x^{* *} \oplus y^{* *}$.

(iii). $x \oplus 0=\left(x^{*} \otimes 0^{*}\right)^{*} \stackrel{(C 10)}{=} x^{* *}$.

(iv). By (15), $\left(\mathrm{c}_{9}\right),\left(\mathrm{c}_{5}\right)$ and point $(i i)$, we have

$x \oplus(y \oplus z) \stackrel{(15)}{=} x^{*} \rightarrow\left(z^{*} \rightarrow y^{* *}\right)^{* *}=x^{*} \rightarrow(z \oplus y)^{* *}=x^{*} \rightarrow(z \oplus y) \stackrel{(15)}{=} x^{*} \rightarrow\left(z^{*} \rightarrow y^{* *}\right) \stackrel{(C 5)}{=}\left(x^{*} \otimes z^{*}\right) \rightarrow y^{* *}$ and

$(x \oplus y) \oplus z^{(i),(15)}=z^{*} \rightarrow\left(x^{*} \rightarrow y^{* *}\right)^{* *} \stackrel{(15)}{=} z^{*} \rightarrow(x \oplus y)^{* *} \stackrel{(i i)}{=} z^{*} \rightarrow(x \oplus y) \stackrel{(15)}{=} z^{*} \rightarrow\left(x^{*} \rightarrow y^{* *}\right) \stackrel{(15)}{=}\left(x^{*} \otimes z^{*}\right) \rightarrow y^{* *}$, hence our claim holds.

(v). $x \oplus 1 \stackrel{(15)}{=} x^{*} \rightarrow 1^{* *} \stackrel{(C 10)}{=} x^{*} \rightarrow 1 \stackrel{(C 2)}{=} 1$. 
(vi). Since $x \leq x^{* *}, y \leq y^{* *}$ and $x^{* *}, y^{* *} \leq x \oplus y$ implies $x \vee y \leq x \oplus y$. Thus,

$1=x \vee y \leq x \oplus y \Rightarrow x \oplus y=1$.

(vii). By $\left(\mathrm{c}_{7}\right),\left(\mathrm{c}_{10}\right)$ and $\left(\mathrm{c}_{9}\right)$, we obtain successively

$$
\begin{aligned}
& x \oplus(y \vee z)^{*} \stackrel{(15)}{=} x^{*} \rightarrow(y \vee z)^{* * * *}=x^{*} \rightarrow(y \vee z)^{*} \stackrel{(C 9)}{=} x^{*} \rightarrow\left(y^{*} \wedge z^{*}\right) \stackrel{(C 7)}{=}\left(x^{*} \rightarrow y^{*}\right) \wedge\left(x^{*} \rightarrow z^{*}\right) \stackrel{(C 9)}{=} \\
& \left(y^{* *} \rightarrow x^{* *}\right) \wedge\left(z^{* *} \rightarrow x^{* *}\right) \stackrel{(15)}{=}\left(x \oplus y^{*}\right) \wedge\left(x \oplus z^{*}\right) .
\end{aligned}
$$

(viii). We obtain successively

$x \oplus\left(y^{*} \vee z^{*}\right)^{*}=x^{*} \rightarrow\left(y^{*} \vee z^{*}\right)^{* * * *}=x^{*} \rightarrow\left(y^{*} \vee z^{*}\right)^{*}=x^{(C 9)} \rightarrow\left(y^{* *} \wedge z^{* *}\right) \stackrel{(C 7)}{=}\left(x^{*} \rightarrow y^{* *}\right) \wedge\left(x^{*} \rightarrow z^{* *}\right) \stackrel{(15)}{=}$ $(x \oplus y) \wedge(x \oplus z)$.

$(i x) . x \oplus x^{*} \stackrel{(i)}{=} x^{*} \oplus x \stackrel{(15)}{=} x^{* *} \rightarrow x^{* *} \stackrel{(C 2)}{=} 1$.

$(x) .\left(x^{*} \oplus y\right)^{*} \oplus y \stackrel{(15)}{=}\left(x^{* *} \rightarrow y^{* *}\right)^{*} \oplus y \stackrel{(15)}{=}\left(x^{* *} \rightarrow y^{* * * * *} \rightarrow y^{* *}\right.$

and $\left(y^{*} \oplus x\right)^{*} \oplus x=\left(y^{* * *} \rightarrow x^{* * *}\right)^{*} \oplus x=\left(y^{* * *} \rightarrow x^{* *}\right)^{* *} \rightarrow x^{* *}$. Thus, our claim holds.

For $x \in L$ and $n \geq 0$, we define $0 \cdot x=0$ and $n x=(n-1) x \oplus x$.

Corollary 2 If $x, y \in L$ and $m, n \geq 1$, then

(i) If $m \leq n$, then $m x \leq n x$;

(ii) If $x \leq y$, then $m x \leq m y$;

Proof. (i). Since $x \stackrel{(10)}{\leq} x^{* *} \Rightarrow x \stackrel{(C 2)}{\leq} x^{*} \rightarrow x^{* *} \stackrel{(15)}{=} x \oplus x=2 x$, we deduce that $x \leq 2 x$, that is, if $m \leq n$, then $m x \leq n x$, for any natural numbers $m, n \geq 1$.

(ii). Since $x \leq y$ we obtain successively $y^{*} \stackrel{(C 4)}{\leq} x^{*}, \quad y^{*} \otimes y^{*} \stackrel{(C 3)}{\leq} x^{*} \otimes x^{*}$,

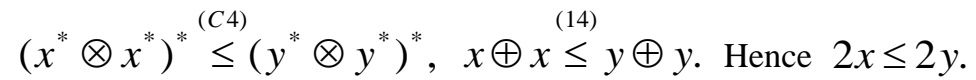

By induction, we deduce that $m x \leq m y$, for every natural number $m \geq 1$.

Lemma 1 Let $L$ be a residuated lattice and $x, y, z \in L$.

Then:

$\left(c_{11}\right)$ If $x \leq y$, then $x \oplus z \leq y \oplus z$.

Proof. $\left(c_{11}\right)$. If $x \leq y$ we obtain successively $y^{*} \leq x^{*}, \quad x^{*} \rightarrow z^{* *} \leq y^{*} \rightarrow z^{* *}$. Hence $x \oplus z \leq y \oplus z$.

Corollary 3 ([8]) Let $L$ be a Stonean residuated lattice. If $x, y \in L$ and $n \geq 2$, then

$\left(c_{12}\right) \quad x \wedge(n y) \leq n(x \otimes y)$.

Proof. Mathematical induction relative to $n$.

Corollary 4 ([8]) Let $L$ be a Stonean residuated lattice. If $x, y \in L$ and $m \geq 2$ or $n \geq 2$, then $\left(c_{13}\right)(m x) \wedge(n y) \leq(m n)(x \otimes y)$.

Proof. Suppose $m \geq 2$. If $n=0$ in $\left(c_{13}\right)$ we have equality. If $n=1,\left(c_{13}\right)$ follows from $\left(c_{12}\right)$. 
If $n \geq 2$, by $\left(c_{12}\right)$ we deduce that $(m x) \wedge(n y) \leq n[(m x) \wedge y] \leq n[m(x \otimes y)]=(m n)(x \otimes y)$. Analogously, if $n \geq 2$.

Corollary 5 ([8]) Let $L$ be a Stonean residuated lattice. If $x, y \in L$ and $m, n$ are natural numbers, then $\left(c_{14}\right) \quad(m x) \otimes(n y) \leq(m n)(x \otimes y) ;$

$\left(c_{15}\right)(m x) \wedge(n y) \leq(m n)(x \wedge y)$.

Proof. $\left(c_{14}\right)$. If $m=0$, then in $\left(c_{14}\right)$ we have equality for every natural number $n$.

If $m=1$ and $n=0,1$, we have also equality in $\left(c_{14}\right)$. If $m=1$ and $n \geq 2$, then we have $x \otimes(n y) \leq x \wedge(n y) \stackrel{\left(c_{12}\right)}{\leq} n(x \otimes y)$.

If $m \geq 2$, then $(m x) \otimes(n y) \leq(m x) \wedge(n y) \stackrel{\left(c_{13}\right)}{\leq}(m n)(x \otimes y)$.

$\left(c_{5}\right)$. As in the case of $\left(c_{14}\right)$, using $\left(c_{13}\right)$.

Following the paper [30] we extend the notion of filter's radical to residuated lattices, as $i$-filter's radical. Initially, the filter's radicals were defined and studied in lattice implication algebras (see [30]), we extend this notion to residuated lattices as i-filter's radicals and we present some specific properties of them in Stonean residuated lattices.

Definition 6 Let $D \in \mathrm{F}_{i}(L)$ be an i-filter of $L$. The subset

$\{x \in L:$ thereis $n \in N$ such that $n x \in D\}$

is called an i-filter's radical, and it is denoted by $\sqrt{D}$.

In [30], the authors define a lattice implication algebra as follows:

Definition 7 ([30]) By a lattice implication algebra we mean a bounded lattice $(L, \wedge, \vee, 0,1)$ with order-reversing involution 1 and 0 the greatest and the smallest element of $L$ respectively, and a binary operation $\rightarrow$ satisfying the follow axioms:

$\left(I_{1}\right) x \rightarrow(y \rightarrow z)=y \rightarrow(x \rightarrow z)$;

$\left(I_{2}\right) \quad x \rightarrow x=1$;

$\left(I_{3}\right) x \rightarrow y=y^{*} \rightarrow x^{*}$

$\left(I_{4}\right)$ If $x \rightarrow y=y \rightarrow x=1$, then $x=y$;

$\left(I_{5}\right)(x \rightarrow y) \rightarrow y=(y \rightarrow x) \rightarrow x$;

$\left(L_{1}\right) \quad(x \vee y) \rightarrow z=(x \rightarrow z) \wedge(y \rightarrow z)$;

$\left(L_{2}\right) \quad(x \wedge y) \rightarrow z=(x \rightarrow z) \vee(y \rightarrow z)$

for all $x, y, z \in L$.

Corollary 6 There are Stonean residuated lattices which are not lattice implication algebras, so, it is proper to study i-filter's radicals in Stonean residuated lattices.

Proof. In Example 3 we have $0^{*} \vee 0^{* *}=1,1^{*} \vee 1^{* *}=1, a^{*} \vee a^{* *}=f \vee b=1, b^{*} \vee b^{* *}=f \vee b=1$, $c^{*} \vee c^{* *}=b \vee f=1, d^{*} \vee d^{* *}=0 \vee 1=1, e^{*} \vee e^{* *}=0 \vee 1=1, f^{*} \vee f^{* *}=b \vee f=1$, $g^{*} \vee g^{* *}=0 \vee 1=1$, that is, $L$ is Stonean.

We consider the Stonean residuated lattice from Example 3, where $(a \rightarrow f) \rightarrow f=f \rightarrow f=1$ and $(f \rightarrow a) \rightarrow a=b \rightarrow a=g$, so, $(a \rightarrow f) \rightarrow f=1 \neq g=(f \rightarrow a) \rightarrow a$, that is, the axiom $\left(I_{5}\right)$ from Definition 7 is not satisfied. 
Remark 6 In a residuated lattice $L$, an i-filter's radical is not always an $i$-filter.

Indeed, see Example 2, for $D=\{1\}$ we have $\sqrt{D}=\{1, e, g, d\}$.

By Remark 2, we deduce that $\sqrt{D}$ is not an i-filter.

Following Remark 6, we deduce that an i-filter's radical is not closed with respect to the operation e.

Remark 7 The residuated lattice from Example 2 is not Stonean.

Indeed, the Example 2 is not Stonean, because $d^{*} \vee d^{* *}=d \neq 1$.

Proposition 7 Let $D \in \mathrm{F}_{i}(L)$ be an i-filter of a residuated lattice $L$. Then $D \subseteq \sqrt{D}$.

Proof. Let $D \in \mathrm{F}_{i}(L)$ be an i-filter of $L$ and $x \in D$. Since $D$ is an i-filter and $x \in D, x \leq x \oplus x$, we deduce that $x \oplus x=2 x \in D$, that is, $x \in \sqrt{D}$.

Lemma 2 Let $D \in \mathrm{F}_{i}(L)$ be an $i$-filter of a residuated lattice L. Then:

(i) $1 \in \sqrt{D}$;

(ii) If $x \in \sqrt{D}$ and $x \leq y$, then $y \in \sqrt{D}$;

(iii) If $x, y \in \sqrt{D}$, then $x \rightarrow y \in \sqrt{D}$.

Proof. (i). Since for every i-filter $D \in \mathrm{F}_{i}(L)$ we have $1 \in D$, and $n \cdot 1=1$, for every $n \geq 1$, we deduce that $1 \in \sqrt{D}$.

(ii). If $x \in \sqrt{D}$, then there exists $n \geq 1$ such that $n x \in D$. Because $x \leq y$, by Corollary 2, (ii) we have $n x \leq n y$, since $D$ is an i-filter and $n x \in D, n x \leq n y$, then $n y \in D$, that is, $y \in \sqrt{D}$.

(iii). If $x, y \in \sqrt{D}$, then there exist $m, n \geq 1$ such that $m x \in D$ and $n y \in D$. Since (C2)

$m x \in D, m x \leq m(x \rightarrow y)$, we deduce that $m(x \rightarrow y) \in D$, that is, $x \rightarrow y \in \sqrt{D}$.

Following Lemma 2 we conclude that:

Theorem 2 In any residuated lattice $L$, the i-filter's radicals are lattice filters closed with respect to the operation $\rightarrow$.

Since in any residuated lattice $L$, the i-filter's radicals are lattice filters, then they are closed with respect to the operation $\wedge$. In the case of Stonean residuated lattices we establish that:

Lemma 3 Let $D \in \mathrm{F}_{i}(L)$ be an i-filter of a Stonean residuated lattice L. Then $x, y \in \sqrt{D}$ if, and only if, $x \wedge y \in \sqrt{D}$.

Proof. Let $D \in \mathrm{F}_{i}(L)$. Assume that $x \in \sqrt{D}$ and $y \in \sqrt{D}$, then there exist $m, n \geq 1$ such that $m x \in D$ and $n y \in D$. Without restricting the generality we can assume that $m \leq n$. By Corollary 2, $(i)$ we have $m x \leq n x$. Since $D \in \mathrm{F}_{i}(L)$ is an i-filter of $L$ and $m x \in D$, then $n x \in D$.

By (7), we have $n x \rightarrow(n x \wedge n y) \stackrel{(C 7)}{=}(n x \rightarrow n x) \wedge(n x \rightarrow n y) \stackrel{(C 2)}{=} n x \rightarrow n y$. Since $n x \rightarrow n y \in D$, we deduce that $n x \rightarrow(n x \wedge n y) \in D$. Since $D$ is an i-filter and $n x \in D, n x \rightarrow(n x \wedge n y) \in D$, then $n x \wedge n y \in D$. Following Corollary $4,\left(c_{7}\right)$ we have $n x \wedge n y \leq\left(n^{2}\right)(x \wedge y) \in D$, then we deduce that $x \wedge y \in \sqrt{D}$.

Conversely, if $x \wedge y \in \sqrt{D}$, then there exists $n \geq 1$ such that $n(x \wedge y) \in D$. By Corollary 2, (ii) we have 
$n(x \wedge y) \leq n x, n y$, and since $D$ is an i-filter, then $n x \in D$ and $n y \in D$, that is, $x, y \in \sqrt{D}$.

Following Lemma 3 we conclude that an i-filter's radical in a Stonean residuated lattice is closed relative to the operation $\wedge$.

Theorem 3 Let $P, Q \in \mathrm{F}_{i}(L)$ be two i-filters of a residuated lattice $L$. Then

(i) If $P \subseteq Q$, then $\sqrt{P} \subseteq \sqrt{Q}$;

(ii) $\sqrt{P} \cap \sqrt{Q}=\sqrt{P \cap Q}$;

(iii) $\sqrt{P} \subseteq \sqrt{\langle\sqrt{P}\rangle}$.

Proof. (i). We notice that for all $x \in \sqrt{P}$ there exists $n \geq 1$ such that $n x \in P \subseteq Q$. Therefore, $x \in \sqrt{Q}$, that is, $\sqrt{P} \subseteq \sqrt{Q}$.

(ii). Clearly, $P \cap Q$ is an i-filter of $L$. Since $P \cap Q \subseteq P$ and $P \cap Q \subseteq Q$, by (i) we have $\sqrt{P \cap Q} \subseteq \sqrt{P} \cap \sqrt{Q}$.

Conversely, for any $x \in \sqrt{P} \cap \sqrt{Q}$ we have $x \in \sqrt{P}$ and $x \in \sqrt{Q}$, so, there exist $m, n \geq 1$ such that $m x \in P$ and $n x \in Q$. Clearly, by Corollary 2, (i) we deduce that $(m+n) x \in P \cap Q$. Therefore, $x \in \sqrt{P \cap Q}$.

(iii). Following Proposition 7 we have $P \subseteq \sqrt{P}$, by $(i)$, we deduce that $\sqrt{P} \subseteq \sqrt{\langle\sqrt{P}\rangle}$.

Following the properties of a principal i-filter from Proposition 3 we obtain:

Corollary 7 Let $L$ be a residuated lattice. Then

(i) If $a \leq b$, then $\sqrt{\langle b\rangle} \subseteq \sqrt{\langle a\rangle}$;

(ii) $\sqrt{\langle a\rangle \cap\langle b\rangle}=\sqrt{\langle a \vee b\rangle}$;

(iii) If $a \vee b=1$, then $\sqrt{\langle a\rangle \cap\langle b\rangle}=\sqrt{\{1\}}$.

Proof. (i). and (ii). are obvious.

(iii). By (ii), we deduce that $\sqrt{\langle a\rangle \cap\langle b\rangle}=\sqrt{\{1\}}$.

Lemma 4 Let $P$ be a prime i-filter of a residuated lattice L. If $\sqrt{P}$ is an i-filter of $L$, then not always $\sqrt{P}$ is prime.

Proof. We consider the Example 1, where $P=\{1\}$ is a prime i-filter and its i-filter's radical is $\sqrt{P}=\{1, c\}$. Clearly, $\{c, 1\},\{a, c, 1\}$ and $\{b, c, 1\}$ are i-filters of $L$.

Following Proposition 4, since $\sqrt{P}=\{1, c\}=\{a, c, 1\} \cap\{b, c, 1\}$ with $\{1, c\} \neq\{a, c, 1\}$ and $\{1, c\} \neq\{b, c, 1\}$, we deduce that $\sqrt{P}$ is not a prime i-filter.

As a second proof, we can follow the Remark 3, since $a \vee b=c \in \sqrt{P}$ with $a \notin \sqrt{P}$ and $b \notin \sqrt{P}$, then $\sqrt{P}$ is not a prime i-filter.

We say that a residuated lattice has the double negation property if for all $x \in L$ hold $x^{* * *}=x$.

Remark 8 Let $P$ be a prime i-filter of a G-algebra $L$ with double negation property. If $\sqrt{P}$ is an i-filter of 
$L$, then $\sqrt{P}$ is prime.

Indeed, it is well known that in a BL-algebra $L$ we have the following rule of calculus: $(x \wedge y)^{*}=x^{*} \vee y^{*}$. Since

$2(x \vee y)=(x \vee y) \oplus(x \vee y) \stackrel{(14)}{=}\left[(x \vee y)^{*} \otimes(x \vee y)^{*}\right]^{*} \stackrel{(13)}{=}(x \vee y)^{* * *} \stackrel{(C 9)}{=}\left(x^{*} \wedge y^{*}\right)^{*}=x^{* *} \vee y^{* *}=x \vee y$ and $2 x=x \oplus x=x^{* *}=x$, we deduce that $n(x \vee y)=n x \vee n y$, for any $n \geq 1$.

In order to prove that $\sqrt{P}$ is prime, we consider $x, y \in L$ such that $x \vee y \in \sqrt{P}$ and $x \notin \sqrt{P}$. Then there exists $n \geq 1$ such that $n(x \vee y) \in P$. Since $P$ is prime and $n(x \vee y)=n x \vee n y \in P$ and $n x \notin P$, we deduce that $n y \in P$, that is, $y \in \sqrt{P}$. So, $\sqrt{P}$ is prime.

Proposition 8 ([8]) If $L$ is a Stonean residuated lattice then for every $x, y \in L$ :

$\left(c_{16}\right)(x \wedge y)^{*}=x^{*} \vee y^{*}$.

Lemma 5 Let $L$ a Stonean residuated lattice. If $M \in \operatorname{Max}_{i}(L)$ is a maximal $i$-filter of $L$, then $M=\sqrt{M}$.

Proof. By maximality of $M$, it is sufficient to prove that $\sqrt{M} \in \mathrm{F}_{i}(L)$ is an i-filter of $L$.

For that, we consider $x, y \in \sqrt{M}$. Then there exist $m, n \geq 1$ such that $m x \in M$ and $n y \in M \Rightarrow(m x) \otimes(n y) \in M$. Following $\quad\left(c_{14}\right) \quad$ we deduce that $(m n)(x \otimes y) \in M$, that is, $x \otimes y \in \sqrt{M}$. Thus, $\sqrt{M} \in \mathrm{F}_{i}(L)$.

The following results (Theorem 4, Theorem 5) represent new characterizations for maximal i-filters in Stonean residuated lattices.

Theorem 4 Let $L$ be a Stonean residuated lattice and $a \in L$. Then the following assertions are equivalent:

(i) $M \in \operatorname{Max}_{i}(L)$

(ii) $M \in \operatorname{Spec}_{i}(L), \quad x \in M$ iff $x^{* *} \in M$

Proof. $(i) \Rightarrow$ (ii). Clear by Remarks 4 and 5 .

(ii) $\Rightarrow(i)$. Let $x \notin M$. Then $x^{* *} \notin M$. Since $L$ is a Stonean residuated lattice we have $x^{*} \vee x^{* *}=1$. Since $M \in \operatorname{Spec}_{i}(L)$ and $x^{*} \vee x^{* *}=1 \in M, x^{* *} \notin M$, then $x^{*} \in M$.

If $x^{*} \in M$. Since $x^{n} \leq x$, for every $n \geq 1$, then $x^{*} \stackrel{(C 4)}{\leq}\left(x^{n}\right)^{*}$. Since $M$ is an i-filter we deduce that $\left(x^{n}\right)^{*} \in M$.

If for any $x \in L,\left(x^{n}\right)^{*} \in M$, for some $n \geq 1$, then $x \notin M$. If by contrary $x \in M$, then $x^{n} \in M$, for every $n \geq 1$, and $x^{n} \otimes\left(x^{n}\right)^{*}=0 \in M$, a contradiction.

We deduce that for any $x \in L, \quad x \notin M$ iff $\left(x^{n}\right)^{*} \in M$, for some $n \geq 1$, that is, $M \in \operatorname{Max}_{i}(L)$ (see Corollary 1, (ii) ).

Theorem 5 Let $M \in \operatorname{Spec}_{i}(L)$ be a proper i-filter of a Stonean residuated lattice L. Then $M=\sqrt{M}$ if, and only if, $M \in \operatorname{Max}_{i}(L)$.

Proof. Following Lemma 5, we deduce that if $M \in \operatorname{Max}_{i}(L)$, then $M=\sqrt{M}$.

Conversely, if $M=\sqrt{M}$, then $\sqrt{M} \in F_{i}(L)$ is an i-filter of $L$. Thus, by $\left(\mathrm{c}_{10}\right)$, if $x \in \sqrt{M}$, then $x^{* *} \in \sqrt{M}$. If $x^{* *} \in \sqrt{M}$, then there is $n \geq 1$ such that 
$n x^{* *} \in M \stackrel{(C 2)}{\Rightarrow} n\left(x^{*} \rightarrow x^{* *}\right) \in M \stackrel{(14)}{\Rightarrow} n(2 x) \in M \Rightarrow(2 n) x \in M$, that is, $x \in \sqrt{M}$.

Therefore, $x \in \sqrt{M}$ iff $x^{* *} \in \sqrt{M}$, following Theorem 4 we deduce that $\sqrt{M} \in \operatorname{Max}_{i}(L)$. Thus,

$M \in \operatorname{Max}_{i}(L)$.

Lemma 6 Let $L$ be a non-degenerate residuated lattice. If $M=\sqrt{M}$, then $M \in \operatorname{Max}_{i}(L)$.

Proof. See the proof $(" \Rightarrow ")$ of Theorem 5.

We propose to solve the following exercise.

Remark 9 On a given Stonean residuated lattice $L$, show that: If $M \in \operatorname{Max}_{i}(L)$, then $M=\sqrt{M}$.

Indeed, if we choose the Example 3. For any natural number $n \geq 1$ we have

$n 1=1, n g=1, n f=f, n e=1, n d=1, n c=f, n b=b, n a=b$. Clearly, $\langle a\rangle=\{a, b, d, e, g, 1\}$ and

$\langle c\rangle=\{c, d, f, e, g, 1\}$ are the maximal i-filter of $L$. It is easy to see that $\langle a\rangle=\sqrt{\langle a\rangle}$ and $\langle c\rangle=\sqrt{\langle c\rangle}$.

Remark 10 In a Stonean residuated lattice $L$, if $\sqrt{M} \in \operatorname{Max}_{i}(L)$ is a maximal i-filter of $L$, then not always $\sqrt{M}=M$.

Indeed, for that we consider the Example 3. Clearly, $\langle b\rangle=\{b, e, 1\}$ is an i-filter of $L$ and $\sqrt{\langle b\rangle}$

$=\{a, b, d, e, g, 1\}=\langle a\rangle$ is a maximal i-filter of $L$. Thus, $\sqrt{\langle b\rangle} \in \operatorname{Max}_{i}(L)$ and $\sqrt{\langle b\rangle} \neq\langle b\rangle$.

Definition 8 Let $L_{1}$ and $L_{2}$ be two residuated lattices and $f: L_{1} \rightarrow L_{2}$ be a mapping from $L_{1}$ to $L_{2}$.

We call $f$ a morphism of residuated lattices if for all $x, y \in L_{1}$ it satisfies:

$\left(M_{1}\right) \quad f(0)=0$ and $f(1)=1$;

$\left(M_{2}\right) \quad f(x \wedge y)=f(x) \wedge f(y)$

$\left(M_{3}\right) \quad f(x \vee y)=f(x) \vee f(y)$

$\left(M_{4}\right) \quad f(x \otimes y)=f(x) \otimes f(y)$

$\left(M_{5}\right) \quad f(x \rightarrow y)=f(x) \rightarrow f(y)$;

$\left(M_{6}\right) \quad f\left(x^{*}\right)=(f(x))^{*}$.

Lemma 7 Let $L_{1}$ and $L_{2}$ be two residuated lattices. If $f: L_{1} \rightarrow L_{2}$ is a morphism of residuated lattices, then $f(n x)=n f(x)$, for all $x \in L_{1}$ and every natural number $n \geq 1$.

Proof. Since $f$ is a morphism of residuated lattices, then $f(x \oplus x) \stackrel{(14)}{=} f\left(\left(x^{*} \otimes x^{*}\right)^{*}\right) \stackrel{\left(M_{6}\right),\left(M_{4}\right)}{=}$

$\left(f\left(x^{*} \otimes x^{*}\right)\right)^{*}=\left(f\left(x^{*}\right) \otimes f\left(x^{*}\right)\right)^{*} \stackrel{\left(M_{6}\right)}{=}\left(f(x)^{*} \otimes f(x)^{*}\right)^{*} \stackrel{(14)}{=} f(x) \oplus f(x)$, by the associativity of $\oplus$ (see Proposition 6, (iv) ), we deduce that $n f(x)=f(n x)$, for all $x \in L_{1}$ and every natural number $n \geq 1$.

Let $f$ be a morphism of residuated lattices from $L_{1}$ to $L_{2}$ and $1_{L_{2}}$ be the greatest element of $L_{2}$. We define the dual kernel of $f$ denoted by $D-\operatorname{ker}(f)$, as

$$
D-\operatorname{ker}(f):=\left\{x \in L_{1}: f(x)=1_{L_{2}}\right\} .
$$

Theorem 6 Let $f: L_{1} \rightarrow L_{2}$ be an onto morphism of residuated lattices. If $D \in \mathrm{F}_{i}\left(L_{1}\right)$ is a proper $i$-filter of $L_{1}$, and $D-k e r(f) \subseteq D$, then $f(\sqrt{D})=\sqrt{f(D)}$. 
Proof. Clearly, $f(D) \in \mathrm{F}_{i}\left(L_{2}\right)$ is a proper i-filter of $L_{2}$.

Firstly, we prove that $f(\sqrt{D}) \subseteq \sqrt{f(D)}$.

For that we consider $y \in f(\sqrt{D})$, then there exists $x \in \sqrt{D}$ such that $f(x)=y$. Since $x \in \sqrt{D}$, then there is $n \geq 1$ such that $n x \in D$. Following Lemma 7 we have $f(n x)=n f(x)=n y \in f(D)$, that is, $y \in \sqrt{f(D)}$.

Conversely, we consider $y \in \sqrt{f(D)}$, then there is $n \geq 1$ such that $n y \in f(D)$. Since $D$ is morphism of residuated lattices, then there exists $x \in L_{1}$ such that $f(x)=y$. Following Lemma 7 we have $f(n x)=n f(x)=n y$, and by the fact that $f$ is onto, we deduce that there exists $z \in D$ such that $f(n x)=f(z)$. It follows that $f\left(z \rightarrow_{1} n x\right)=f(z) \rightarrow_{2} f(n x)=1_{L_{2}}$, that is, $z \rightarrow_{1} n x \in D-k e r(f)$.

Because $D-\operatorname{ker}(f) \subseteq D \Rightarrow z \rightarrow{ }_{1} n x \in D \Rightarrow n x \in D$, that is, $x \in \sqrt{D}$. Therefore, $y=f(x) \in f(\sqrt{D})$.

In what follows we present an easy but important consequence of Theorem 6:

Corollary 8 Let $f: L_{1} \rightarrow L_{2}$ be an onto morphism of residuated lattices. Then:

(i) If $\sqrt{D} \in \mathrm{F}_{i}\left(L_{1}\right)$ and $D-k e r(f) \subseteq D$, then $\sqrt{f(D)} \in \mathrm{F}_{i}\left(L_{2}\right)$;

(ii) If $\sqrt{D} \in \operatorname{Spec}_{i}\left(L_{1}\right)$ and $D-\operatorname{ker}(f) \subseteq D$, then $\sqrt{f(D)} \in \operatorname{Spec}_{i}\left(L_{2}\right)$.

In what follows we present an easy but important consequence of Theorem 5 and Theorem 6:

Corollary 9 Let $f: L_{1} \rightarrow L_{2}$ be an onto morphism of Stonean residuated lattices. If $D \in \operatorname{Max}_{i}\left(L_{1}\right)$ and $D-\operatorname{ker}(f) \subseteq D$, then $\sqrt{f(D)} \in \operatorname{Max}_{i}\left(L_{2}\right)$.

Proof. Obvious, by Theorem 4.

Theorem 7 Let $f: L_{1} \rightarrow L_{2}$ be a morphism of residuated lattices. If $D \in \mathrm{F}_{i}\left(L_{1}\right)$ is a proper i-filter of $L_{1}$, then $f^{-1}(\sqrt{D})=\sqrt{f^{-1}(D)}$.

Proof. Clearly, if $D_{2} \in \mathrm{F}_{i}\left(L_{2}\right)$, then $f^{-1}\left(D_{2}\right) \in \mathrm{F}_{i}\left(L_{1}\right)$.

Firstly, we prove that $f^{-1}(\sqrt{D}) \subseteq \sqrt{f^{-1}(D)}$.

For that we consider $x \in f^{-1}(\sqrt{D})$, then $f(x) \in \sqrt{D}$. Then there exists $n \geq 1$ such that $n f(x) \stackrel{\text { Lemma } 7}{=} f(n x) \in D$. So, $n x \in f^{-1}(D)$, that is, $x \in \sqrt{f^{-1}(D)}$.

Conversely, we consider $x \in \sqrt{f^{-1}(D)}$. Then there is $n \geq 1$ such that $n x \in f^{-1}(D)$, then $f(n x) \stackrel{\text { Lemma } 7}{=}$ $n f(x) \in D$, that is, $f(x) \in \sqrt{D}$. Hence $x \in f^{-1}(\sqrt{D})$. So, $\sqrt{f^{-1}(D)} \subseteq f^{-1}(\sqrt{D})$.

\section{BOOLEAN I-FILTERS IN STONEAN RESIDUATED LATTICES}

An important goal in the residuated lattice theory is to investigate under which conditions every prime i-filter $P \in \operatorname{Spec}_{i}(L)$ is contained in a unique maximal i-filter.

This fact is true for BL-algebras as we can see in what follows. Also, we prove that it is true for Stonean residuated lattice, but it is not true in any residuated lattice. 
Corollary 10 There are residuated lattices in which prime $i$-filters are not contained in a unique maximal $i$-filter.

Proof. We consider Example 4, where $\langle c\rangle=\{c, 1\}$ is prime i-filter which is contained in the maximal i-filters $\langle a\rangle=\{a, n, c, d, 1\}$ and $\langle b\rangle=\{b, n, c, d, 1\}$.

Proposition 9 ([26],Prop. 25,page 19) Let $P$ be a prime i-filter of a BL-algebra $L$. If $D$ is a proper $i$-filter such that $P \subseteq D$, then also $D$ is prime.

Theorem 8 ([26],Th. 3,page 19) In a non-degenerate BL-algebra L, any proper $i$-filter can be extended to a maximal, prime i-filter.

And our goals follow:

Theorem 9 In a non-degenerate BL-algebra $L$, any proper prime $i$-filter $P \in \operatorname{Spec}_{i}(L)$ can be extended to an unique maximal, prime $i$-filter.

Proof. Following Theorem 8 we deduce that $P$ can be extended to a maximal, prime i-filter $M$. It remains to prove the uniqueness.

Supposing by contrary, there are $M_{1}, M_{2} \in \operatorname{Max}_{i}(L)$ two maximal i-filters of $L$ such that $P \subseteq M_{1}$ and $P \subseteq M_{2}$, then $P \subseteq M_{1} \cap M_{2}$. Since the intersection of two i-filters is an i-filter, if we consider $D=M_{1} \cap M_{2}$, then $P \subseteq D$. Following Proposition 9 we deduce that $D$ is a prime i-filter. Following Proposition 4, $(i)$ we deduce that $D=M_{1}$ or $D=M_{2}$, then $P \subseteq M_{1}$ or $P \subseteq M_{2}$, a contradiction.

Theorem 10 ([8]) If $L$ is a Stonean residuated lattice then every prime i-filter is contained in an unique maximal i-filter.

Proof. Let $P$ be a prime i-filter and suppose by contrary that there are two distinct maximal i-filters $M_{1}$ and $M_{2}$ such that $P \subseteq M_{1}$ and $P \subseteq M_{2}$. Since $M_{1} \neq M_{2}$, there is $x \in M_{1}$ such that $x \notin M_{2}$. Following Corollary 1 , (iii), there is $n \geq 1$ such that $\left(x^{n}\right)^{*} \in M_{2}$. Then $\left(x^{n}\right)^{* *} \notin M_{2}$, hence $\left(x^{n}\right)^{* *} \notin P$. Since $x \in M_{1}$, then $x^{n} \in M_{1}$, hence $\left(x^{n}\right)^{*} \notin M_{1}$ and $\left(x^{n}\right)^{*} \notin P$. Since $L$ is supposed Stonean residuated lattice, $\left(x^{n}\right)^{*} \vee\left(x^{n}\right)^{* * *}=1 \in P$, hence $\left(x^{n}\right)^{*} \in P$ or $\left(x^{n}\right)^{* *} \in P$, a contradiction.

Corollary 11 Let $L$ be a Stonean residuated lattice, then $x \wedge x^{*}=0$ for all $x \in L$

Proof. If $x \in L$, then $x^{*} \vee x^{* *}=1$, hence $x^{*} \in B(L)$, so $x^{*} \wedge x^{* *}=0$. Then $x \wedge x^{*} \leq x^{* *} \wedge x^{*}=0$, hence $x \wedge x^{*}=0$.

Proposition 10 For a residuated lattice $L$, the following conditions are equivalent:

(i) $\left(x^{2}\right)^{*}=x^{*}$ for every $x \in L$;

(ii) $x \wedge x^{*}=0$ for every $x \in L$.

Proof. $(i) \Rightarrow\left(\right.$ ii). Let $x \in L$ and $t \in L$ such that $t \leq x, t \leq x^{*}$. Then we obtain successively $t^{2} \leq x \otimes x^{*}=0, \quad t^{2}=0, \quad\left(t^{2}\right)^{*}=0^{*}=1, t^{*}=1, t^{* *}=0$. Since $t^{(C 10)} \leq t^{* * *}$, then $t=0$. Hence $x \wedge x^{*}=0$.

(ii) $\Rightarrow($ i $)$. Let $x \in L$. We obtain successively $x \otimes\left(x \rightarrow x^{*}\right) \leq x \wedge x^{*}=0, \quad x \otimes\left(x \rightarrow x^{*}\right)=0$, $x \rightarrow x^{*} \leq x^{*}, x \rightarrow x^{*}=x^{*}$, then $\left(x^{2}\right)^{*}=x^{*}$. 
If a residuated lattice $L$ satisfies one of the equivalent conditions from Proposition 10, then it is called a SRL-algebra. In ([8]), the following result was proved for Stonean residuated lattices, but it is avaible, also, for SRL-algebras. It is known ([8]) that the class of Stonean residuated lattices is a subclass of SRL-algebras.

Lemma 8 In a SRL-algebra $L$, the following are equivalent:

(i) $\{0\}$ is a prime lattice ideal;

(ii) $x^{*}=0$ for every $x \neq 0$;

(iii) $L \backslash\{0\}$ is a maximal i-filter.

Definition 9 A nonempty subset $F$ of $L$ is called Boolean i-filter if $F$ is an i-filter of $L$ and $x \vee x^{*} \in F$, for every $x \in L$.

Lemma 9 In a Stonean residuated lattice $L$, if there is a Boolean $i$-filter $F$ of $L$ then $F \subseteq R a d(L)$.

Proof. Clearly, the radical of any residuated lattice is an i-filter, because it is the intersection of all maximal i-filters.

Following Proposition 5, for any residuated lattice $L$ we have that

$$
\operatorname{Rad}(L)=\left\{x \in L: \text { for every } n \geq 1 \text {, thereis } \quad k_{n} \geq 1 \text { such that }\left[\left(x^{n}\right)^{*}\right]^{k}=0\right\} .
$$

Following Proposition 10, $(i)$ we deduce that for any Stonean residuated lattice $L$ we have $\left(x^{n}\right)^{*}=x^{*}$, for every $n \geq 1$. Thus, $\operatorname{Rad}(L)=\left\{x \in L:\right.$ there is $k_{n} \geq 1$ such that $\left.\left(x^{*}\right)^{k_{n}}=0\right\}$.

Let $x \in L$. Since $L$ is a SRL-algebra we have $\left(x \vee x^{*}\right)^{*} \stackrel{(C 9)}{=} x^{*} \wedge x^{* *}=0$, that is, $\left(x \vee x^{*}\right) \in \operatorname{Rad}(L)$, for every $x \in L$. Since $F$ is supposed a Boolean i-filter, that is, $x \vee x^{*} \in F$, for every $x \in L$, then we deduce that $F \subseteq \operatorname{Rad}(L)$.

Theorem 11 (Boolean i-filter theorem)

In a Stonean residuated lattice $L$, if there is a prime Boolean i-filter $F$ of $L$, then $F \in \operatorname{Max}_{i}(L)$.

Proof. Following Lemma 9, we deduce that $F \subseteq \operatorname{Rad}(L)$.

Let $x \in \operatorname{Rad}(L)$, then $\left(x^{*}\right)^{n}=0$, for some $n \geq 1$, that is, $x^{*}$ is of finite order, even more there is no i-filter to contain $x^{*}$. Since $F$ is supposed to be a Boolean i-filter, then for every $x \in L, x \vee x^{*} \in F$. Even more, since $x^{*} \notin F, x \vee x^{*} \in F$ and $F$ is supposed to be prime, then $x \in F$, that is,

$\operatorname{Rad}(L) \subseteq F$.

Hence $\operatorname{Rad}(L)=F$.

Because $F$ is prime, then $\operatorname{Rad}(L)$ is prime. Let $\left(M_{i}\right)_{i \geq 1} \in \operatorname{Max}_{i}(L)$ be a sequence of maximal i-filters of $L$. Since $\cap_{i \geq 1} M_{i}=\operatorname{Rad}(L)=F$ and applying successively the Proposition 4 , $(i)$ we deduce that $F=M_{1}$ or $F=M_{2}$ or $\ldots$ or $F=M_{i} \ldots$, that is, $F$ must to be a maximal i-filter.

Corollary 12 In a Stonean residuated lattice $L$, if $L \backslash\{0\}$ is a maximal i-filter, then it is a Boolean i-filter.

Proof. Following Lemma 8, (ii) we deduce that for any $x \in L$ we have $x \vee x^{*}=x \vee 0=x \in L \backslash\{0\}$, hence $x \vee x^{*} \in L \backslash\{0\}$, that is, $L \backslash\{0\}$ is a Boolean i-filter.

\section{ACKNOWLEDGMENT.}

The author was partially supported by the strategic grant POSDRU/CPP107/ DMI1.5/ S/78421, Project ID 78421(2010), co-financed by the European Social Fund - Investing in People, within the Sectorial 
Operational Programme Human Resources Development 2007-2013.

All my gratitude to the annonymious referees for their careful reading of the manuscript and valuable comments which helped in shaping this paper to the present form.

\section{REFERENCES}

[1] R. Balbes, Ph. Dwinger, Distributive Lattices, University of Missouri Press, 1974.

[2] G. Birkhoff, Lattice theory, American Mathematical Society Colloquium Publications, third edition, Providence, 1967.

[3] D. BuȘneag, Categories of Algebraic Logic, Editura Academiei Române, Bucharest, 2006.

[4] D. BuȘneag, D. Piciu, A. Jeflea, Archimedean Residuated Lattices, Annals of the Alexandru Ioan Cuza University - Mathematics, DOI: 10.2478/v10157-010-0017-5, Volume LVI, (2010), 227-252.

[5] C. Bușneag, D. Piciu, The stable topologies for residuated lattices, Soft Computing, 16(2012), 1639-1655.

[6] C. BuȘneag, States and topologies on residuated lattices, Ph D. Thesis, University of Craiova, 2011.

[7] D. Bușneag, D. Piciu and J. Paralescu, Divisible and semi-divisible residuated lattices, Annals of the Alexandru Ioan Cuza University - Mathematics, DOI: 10.2478/aicu-2013-0012, (2013), 14-45.

[8] D. Bușneag, D. Piciu, L.-C. Holdon, Some properties of ideals in Stonean residuated lattices, to appear in Journal of Multiple-Valued Logic and Soft Computing.

[9] L-C. Holdon, L-M. Nițu, G. Chiriac, Distributive residuated lattices, Annals of the University of Craiova - Mathematics and Computer Science Series, Vol. 39(2012), 100-109.

[10] R. L. O. Cignoli, I. M. L. D'Ottaviano and D. Mundici, Algebraic Foundation of Many-Valued Reasoning, Kluwer Academic Publishers, 2000.

[11] R. Cignoli, Free algebras in varieties of Stonean residuated lattices, Soft Computing, 12(2008), 315-320.

[12] R. Cignoli, F. Esteva, Commutative integral bounded residuated lattices with an added involution, Annals of Pure and Applied Logic, 161(2)(2009), 150-160.

[13] H. Freytes, Injectives in residuated algebras, Algebra Universalis, 51 (2004), 373-379.

[14] N. Galatos, P. Jipsen, T. Kowalski, H. Ono, Residuated Lattices: an algebraic glimpse at substructural logics, Studies in Logics and the Foundations of Mathematics, Elsevier, 2007.

[15] V. Glivenko, Sur quelque points de la logique de M. Browwer, Bulletins de l'Academie Royale des Sciences de Belgique, Vol. 15(1929), 183-188.

[16] G. Grätzer, E. T. Schmidt, On a problem of M. H. Stone, Acta Mathematica Academiae Scientiarum Hungarica, Vol. 8, No. 3(1957), 455-460.

[17] G. Grätzer, Lattice Theory: First concepts and distributive lattices, Dover Publications, 2009.

[18] P. Hajek, Mathematics of Fuzzy Logic, Kluwer Academic Publishers Dordrecht, 1998.

[19] A. Iorgulescu, Algebras of logic as BCK algebras, Academy of Economic Studies Bucharest, 2008.

[20] M. Kondo, Simple characterization of strict residuated lattices with an involutive negation, Soft Computing, Vol. 17(2013), 39-44.

[21] T. Kowalski and H. Ono, Residuated lattices: An algebraic glimpse at logics without contraction, Japan Advanced Institute of Science and Technology, (2002), 19-56.

[22] C. Mureșan, The Reticulation of a Residuated Lattice, Bulletin Mathématique de la Société des Sciences Mathématiques de Roumanie, Tome 51(99), No. 1(2008), 47-65.

[23] C. Mureșan, Co-Stone Residuated Lattices, Annals of the University of Craiova - Mathematics and Computer Science Series, Vol. 40(2013), 52-75.

[24] D. Piciu, Algebras of Fuzzy Logic, Editura Universitaria, Craiova, 2007.

[25] M. H. Stone, Topological representations of distributive lattices and Browerian logics, Casopis Pest. Mat. 67(1937), 1-25.

[26] E. Turunen, Mathematics Behind Fuzzy logic, Physica-Verlag Heidelberg, New York, 1999.

[27] J. Varlet, On the characterization of Stone lattices, Acta Scientiarum Mathematicarum (Szeged), Vol. 27(1966), 81-84.

[28] M. Ward, R. P. Dilworth, Residuated lattices, Transactions of the American Mathematical Society, Vol. 45(1939), 335-354.

[29] Y. Zhu, Y. Xu, On filter theory of residuated lattices, Information Science 180(2010), 3614-3632.

[30] H.Zhu, J.B.Zhao, Y.Xu, The filter's radical and the extended filter's in lattice implications algebras, to appear in Iranian Jounal of Fuzzy Systems. 